\title{
Automatic assessment of the quality of G-banded metaphases A preliminary study
}

\author{
CLAES LUNDSTEEN $^{1)}$, JAN MAAHR ${ }^{1)}$ AND TOMMY GERDES ${ }^{2)}$ \\ ${ }^{15}$ Section of Clinical Genetics, Department of Obstetrics and Gynaecology, and \\ ${ }^{2)}$ Department of Biomedical Engineering, Rigshospitalet, \\ Copenhagen, Denmark
}

automation, chromosomes/cytogeneties/quality control

Assessment of the quality of cytogenetic preparations is important for 1) a general quality control of the cytogenetic laboratory and 2) for determining the quality of individual cytogenetic analyses. It is generally accepted that good preparations allow a more detailed search for structural abnormalities than poor preparations.

As part of a comprehensive study on automated assessment of slide quality we have developed a simple algorithm for automated measurement of metaphase resolution. During semi-automated karyotyping with the Magiscan chromosome analysis system (Joyce-Loebl) three measurements related to resolution are automatically extracted 1) the total number of dark bands of the chromosomes of the metaphase (TB), 2) the normalized average length of the chromosomes (NL), and 3) the average "thickness" of the chromosomes $(T)$. The algorithm TB $\times N L / T$ has been tested on 35 metaphases of various quality and compared with visual assessment of the same 35 metaphases. The results indicate that the automatic assessment of metaphase resolution is superior to the visual.

\section{INTRODUCTION}

A basic requirement for a well functioning cytogenetic laboratory is good cytogenetic preparations allowing the cytogeneticist to carry out the analysis with great precision. Thus quality assessment of cytogenetic preparations is required for a general quality control of the cytogenetic laboratory as well as for quality control of individual cases ${ }^{1)}$.

A number of features are available for assessment of the quality of cytogenetic preparations. At low magnification the mitotic index and the overall metaphase quality may be used. At high magnification one may look for quality features associated with the individual metaphase such as resolution, contrast and spreading.

In the past resolution has been evaluated visually, for example by assessment of the number of bands in the haploid chromosome complement, selected chromosomes or chromosome regions by counting or estimation. The UK National External Quality assessment scheme in clinincal cytogenetics ${ }^{2)}$ is based on this procedure and divides the quality of banding into five categories extending from excellent to none. Huret et al. ${ }^{3)}$ proposed to score the quality of banded mitoses on the basis of the number of bands (counted) and length and width (measured by ruler) of chromosome no. 7. Martin et al. ${ }^{4}$ used a detailed scoring sheet for the visual 
evaluation of chromosome slides produced by a semi-automated system for lymphocyte culture, harvest and slide making.

Common for the procedures used so far is that they are more or less based on visual (subjective) assessment by the cytogeneticist and consequently subject to great variation.

During semi-automated karyotyping using chromosome analysis systems like the Magiscan (Joyce-Loebl, U.K.) parameters can easily be extracted which may be related to metaphase quality. Recently we have argued that such parameters may be used to assess the quality of cytogenetic preparation ${ }^{1)}$. In the present study we have measured various parameters during semi-automated karyotyping in order to develop an algorithm for automatic assessment of metaphase resolution. A preliminary test indicates that automatic assessment of resolution is superior to visual assessment.

\section{MATERIALS AND METHODS}

The Magiscan chromosome analysis system for semi-automated karyotyping (Joyce-Loebl, UK) which was used in this study has previously been described in detail ${ }^{5)}$.

Initially we defined a number of parameters which could be extracted from the individual metaphases during semi-automated karyotyping and which we assumed would be related to features like resolution, contrast or spreading. Examples of such parameters are the average length, width and area of the chromosomes of the individual metaphases and the number of dark bands of all chromosome of the metaphases. In this preliminary study we decided to concentrate on parameters which might be used to describe metaphase resolution.

Resolution is not an exactly defined term. It may be argued that resolution reflects the accuracy by which breakpoints may be determined. Resolution is closely related to features such as the number of bands of the chromosomes and the stage of contraction of the chromosomes. Also features such as the width of the chromosomes and "swelling" or "thickness" due to trypsin pretreatment prior to staining may influence the resolution.

15 karyotypes of G-banded metaphases of various quality and from various tissues (peripheral blood, amniotic fluid cells and chorionic villi cultures) were produced with the Magiscan karyotyping system. They were ranked visually according to resolution and the extracted parameters were listed accordingly.

Three of the extracted parameters seemed potentially useful to describe metaphase resolution: 1) the total number of dark bands of all chromosomes of the metaphase (TB), 2) the average normalized length of the chromosomes (NL) and, 3) the "thickness" of the chromosomes $(\mathrm{T})$. TB was defined as the number of peaks of the density profiles of all chromosomes scaled to 32 grey levels ${ }^{6}$. . TB corresponds more or less to the total number of dark bands of the chromosomes. NL was defined as the average normalized length of the measured axes of the chromosomes of the metaphase. A normalization of the length was necessary because the metaphases were digitized at individual secondary magnifications achieved by a motorized zoom mechanism attached to the microscope ${ }^{5)}$. Normalization was obtained by dividing the average lengths of the chromosome axes with the corresponding zoom factor. $T$ was defined as the ratio 
between the mean width and the mean length of the chromosomes, and the mean width was defined as the ratio between the mean area and the mean length of the chromosomes.

From the list of parameters it was noted that in general TB and NL increased with increasing resolution whereas $\mathrm{T}$ in general decreased with increasing resolution. Consequently, it was tentatively decided to use the algorithm: Resolution $=\mathrm{TB} \times \mathrm{NL} / \mathrm{T}$ as an automatic assessment of metaphase resolution.

In order to test this algorithm another $20 \mathrm{G}$-banded metaphases of various quality were karyotyped and for all 35 metaphases the corresponding resolutions were computed using the tentative algorithm allowing the metaphases to be ranked according to their automatically determined resolution.

To test the reproducibility of automatic determination of resolution 5 metaphases were karyotyped twice and their corresponding resolutions were computed.

Five trained cytogeneticists/cytotechnicians (observers) volunteered to rank the 35 karyotypes visually in order to test the algorithm. With an interval of at least 24 hours the five observers ranked the 35 karyotypes twice according to resolution. The observers were instructed carefully not to consider contrast, spreading or other possible features but only the metaphase resolution.

\section{STATISTICAL TESTS}

The Spearman rank-order correlation coefficient $\left(\mathbf{r}_{\mathrm{s}}\right)$ was used to test the relation (agreemen) between 2 sets of ranking (eg. first and second round of ranking by an observer).

With 35 karyotypes (ranked values) the correlation $\left(r_{s}\right)$ would be highly significant if $r_{s}$ was greater than $0.54(\mathrm{p}<0.0005)^{7}$.

The Kendall coefficient of concordance (W) was used to test the relation (agreement) between 5 sets of rankings (eg. the rankings of the five observers in the first round).

With 35 karyotypes (ranked values) and 5 sets of rankings $W$ would be highly significant if $W$ was greater than $0.40(p<0.0005)$. When the relation was significant, the best estimate of the "true" rank for a metaphase would be the average of the 5 ranks".

\section{RESULTS}

Table 1, first column illustrates the reproducibility (agreement) by which each of the five observers ranked the 35 karyotypes twice. The correlation coefficients ( $\left.r_{\mathrm{s}}\right)$ ranged from 0.88 to 0.96 which is highly significant $(\mathrm{p}<\mathbf{0 . 0 0 0 5})$. Fig. 1 illustrates the reproducibility achieved by observer no. 5 .

The agreement $(\mathrm{W})$ between all the five observers within each of the two rounds was also highly significant (round $1 \mathrm{~W}=0.89$; round $2 \mathrm{~W}=0.88 ; \mathrm{p}<0.0005$ ).

The agreement between the observers from round 1 to round 2 is illustrated in Table 1 , second column which shows the correlation coefficients $\left(r_{s}\right)$ for each observer (round 1) 
Table 1. Correlation coefficients ( $r_{s}$ ) obtained in experiments of Visual and automatic ranking of the resolution of 35 karyotypes.

\begin{tabular}{cccc}
\hline Observer & $\begin{array}{c}\text { Within } \\
\text { observer 1) } \\
\mathrm{r}_{\mathrm{s}}\end{array}$ & $\begin{array}{c}\text { Between } \\
\text { observers 2) } \\
\mathrm{r}_{\mathrm{s}}\end{array}$ & $\begin{array}{c}\text { Between machine } \\
\text { and observers 3) } \\
\mathrm{r}_{\mathrm{s}}\end{array}$ \\
\hline 1 & 0.88 & 0.83 & 0.73 \\
2 & 0.91 & 0.96 & 0.94 \\
3 & 0.92 & 0.87 & 0.78 \\
4 & 0.96 & 0.95 & 0.90 \\
5 & 0.95 & 0.94 & 0.93 \\
Average & 0.92 & 0.91 & 0.86 \\
\hline
\end{tabular}

1) Reproducibility of 2 rounds of visual ranking by each of five observers

2) Correlation between visual ranking by each of five observers (round 1) and the average of visual ranking of round 2

3) Correlation between visual ranking by each of five observers (round 1) and automatic ranking

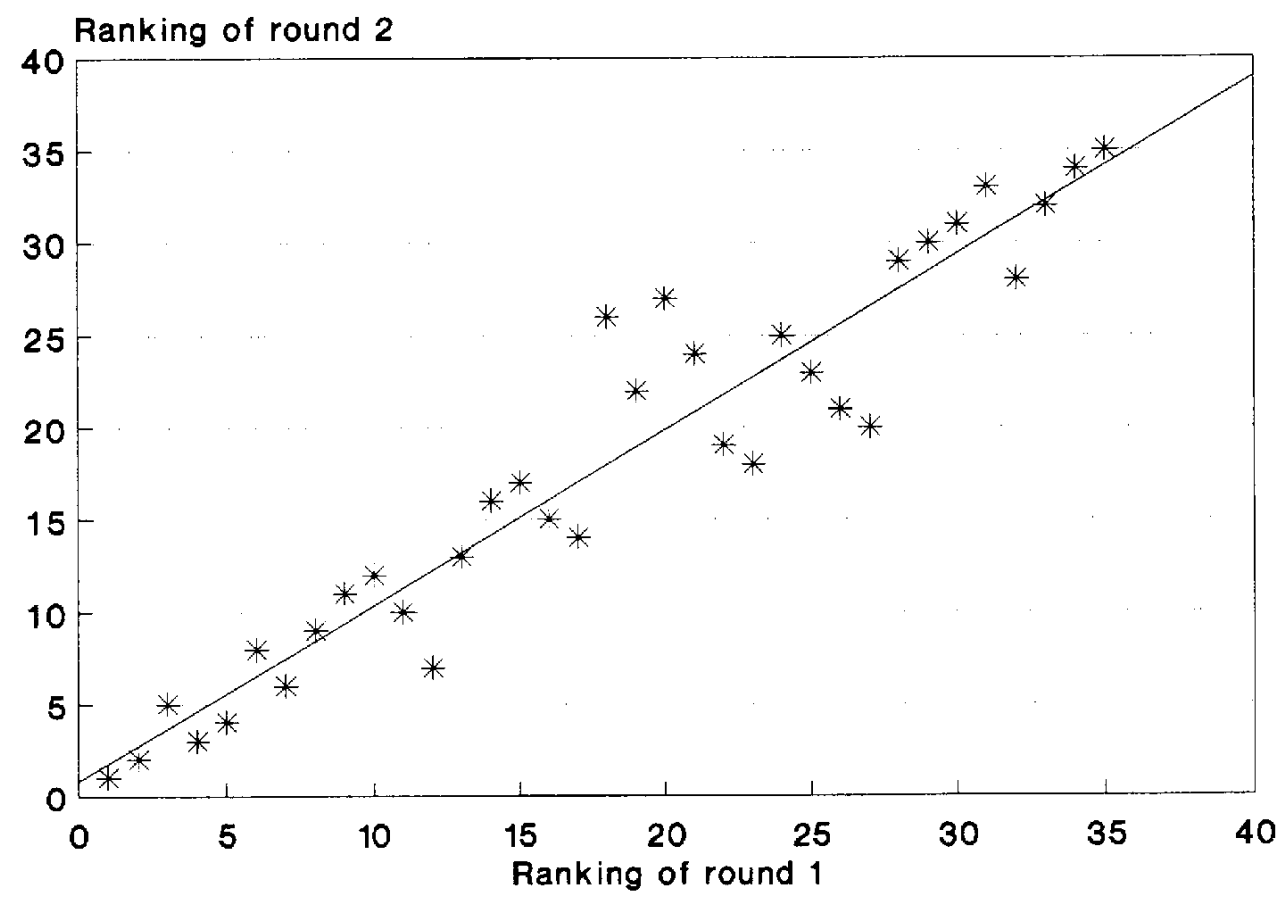

Fig. 1. Correlation between visual ranking of round 1 and 2 for observer 5 .

compared to the average ranking of all five observers in round 2 . The correlation coefficient ranged from 0.83 to $0.96(\mathrm{p}<0.0005)$. Fig. 2 illustrates graphically the correlation between each of the five observers (round 1 ) and the average of round 2 . 
Round 1: Ranks of 5 observers

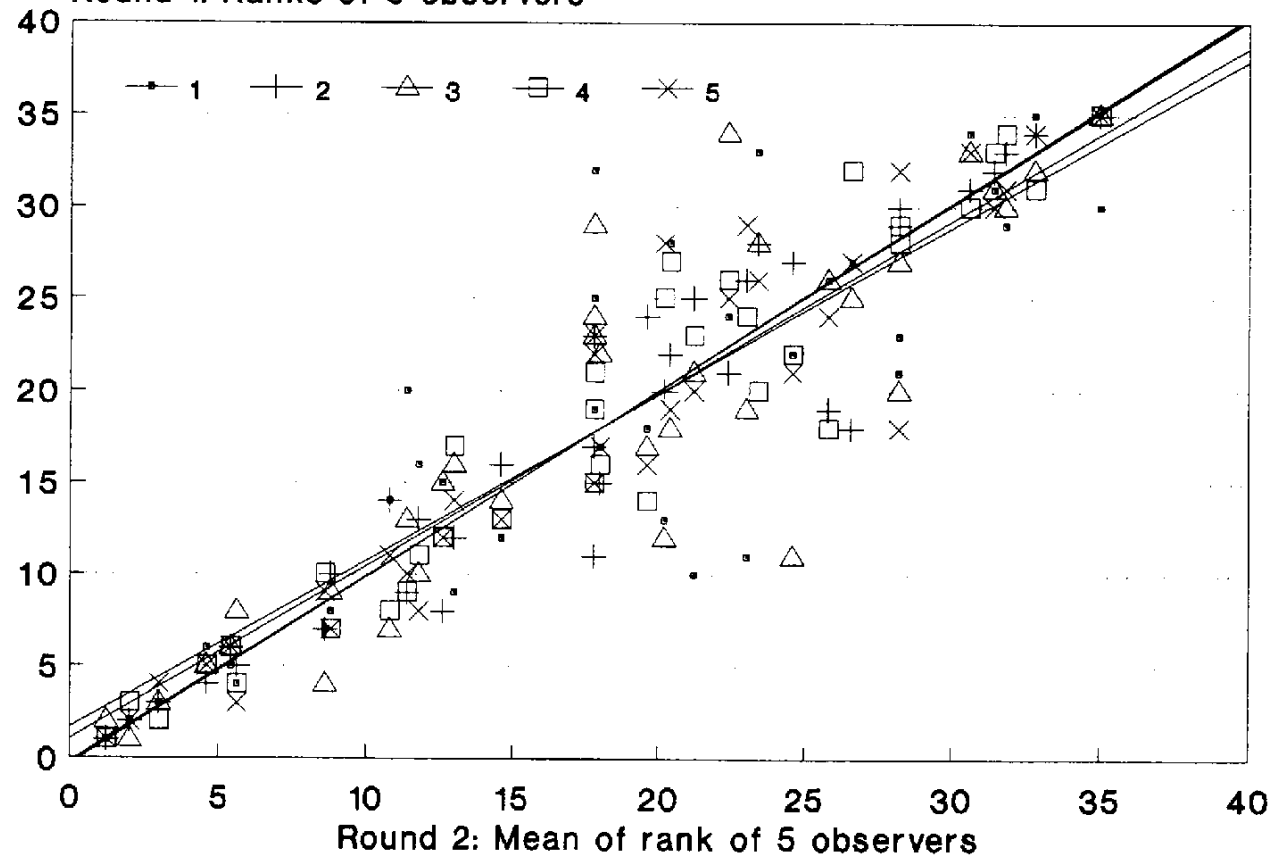

Fig. 2. Correlation between each of 5 observers (round 1) and average of round 2.

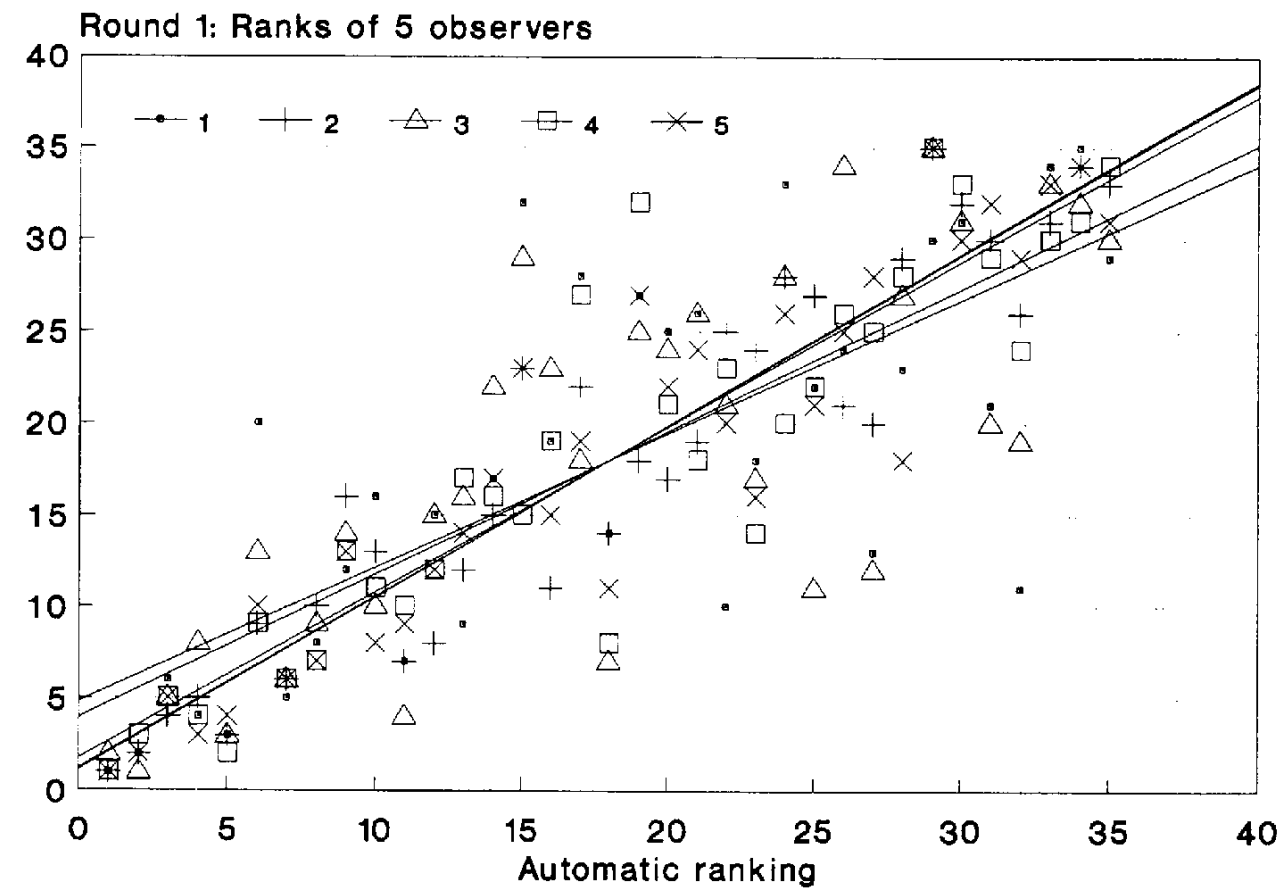

Fig. 3. Correlation between visual ranking by each of five observers (round 1) and atutomatic ranking. 
Table 1, third column shows the agreement between each of the five observers (round 1) and the automatic ranking. The five correlation coefficients $\left(r_{s}\right)$ range from 0.73 to $0.94(p<$ 0.0005 ). The correlations between visual and automatic ranking are illustrated graphically in Fig. 3 .

It appears from table 1 that most of the listed correlation coefficients $\left(r_{s}\right)$ are around 0.90 . In order to provide an example of the confidence limits associated with the computed correlation coefficients we calculated that the confidence interval for a correlation coefficient of 0.90 based on 35 observations ranged between 0.80 and $0.95^{8}$.

The average variation in resolution of 5 metaphases measured automatically twice was $5 \%$.

\section{DISCUSSION}

The purpose of the present study was to develop a fast and reproducible technique for automatic assessment of metaphase resolution to be used along with semi-automated karyotyping.

The results indicate that visual ranking of resolution is reproducible and that there is a good correlation between ranking performed by different observers. In other words, although resolution is a poorly defined feature of a metaphase, and is assumed to be rather subjective, the results show that an observer can repeat the ranking of the resolution of 35 karyotypes with high reproducibility. The results showed also good agreement between the five observers in their ranking of the 35 karyotypes. This was to some extend expected because all five observers are working in the same laboratory with the same kind of cytogenetic preparations. It was, however, not anticipated that the agreement between visual and automatic ranking was as good as the agreement between the five observers. This result indicates that the selected parameters are indeed well suited for an automatic determination of resolution as defined by the algorithm resolution $=\mathrm{TB} \times \mathrm{NL} / \mathrm{T}$.

At this point it should, however, be taken into account that visual ranking requires the presence of all karyotypes at the same time for comparisons. It is easier to determine that one karyotype is "better" than another karyotype than to give the individual karyotype a quality character. Such characters are likely to be influenced by the quality of the previous karyotype and consequently likely to be difficult to reproduce.

The automatic ranking, on the other hand, was based on measurements of individual metaphases. Furthermore, the reproducibility of these measurements was high-only $5 \%$ variation. On that basis it is concluded that automatic assessment of metaphase resolution is: 1) a reliable method for assessing metaphase quality, and 2) a method which is superior to visual assessment.

This conclusion must be taken with some precautions because the experiment was based only on 35 karyotypes of which 15 were also used as learning data. Furthermore, the algorithm may not be useful for more elongated prometaphase or prophase chromosomes.

In the past attempts of defining procedures for objective assessment of metaphase resolution have been rare. Interestingly, Huret et al. ${ }^{3)}$ found that number of bands, length and width 
were suitable parameters for determination of metaphase resolution. These parameters were registered for chromosome 7 and found to correlate well with visual scoring of metaphase quality.

It is worth nothing that the method of Huret et al. ${ }^{3)}$ requires that chromosome 7 is identified before its quality is analysed. A similar procedure might be performed automatically and might provide advantages as compared to the present method. Instead of using the total number of dark bands of all chromosomes and the average length and thickness, one might measure these features on individual automatically classified chromosomes. This procedure might be better than the one used in the present study. An interesting, but so far theoretical, possibility would be to go a step further and to try to identify the individual bands of an automatically classified chromosome on the basis of its density profile. This possibility has previously been discussed ${ }^{9}$.

The procedure for automatic assessment of resolution is intended to be used together with procedures for automatic assessment of contrast and spreading (being developed) for 1) monitoring the general quality of metaphases produced at the laboratory, 2) setting levels for quality required for pre- and postnatal cytogenetics analysis and, 3) for measuring the quality of individual karyotypes being produced at the laboratory.

\section{REFERENCES}

1. Lundsteen, C., Gerdes, T., Maahr, J. (1989) Cytogenetic analysis by automatic multiple cell karyotyping. In "Automation of cytogenetics", Eds. C. Lundsteen and J. Piper, pp. 263-274. Springer-Verlag, Berlin Heidelberg New York.

2. United Kingdom External Quality Assessment Scheme, Slide Assessment Scoring Guide. (1988). ACC Clinical Cytogenetics Bulletin 2: 35-26.

3. Hjuret, J. L., Leonard, C., Aurias, A. (1987) Proposal for a scoring of the quality of the banding of chromosomes. Hum Genet 75: 373-377.

4. Martin, A. O., Shaunnessy, M., Sabrin, H., Maremont, S., Dyer, A., Cimino, M. C., Rissman, A., McKinney, R. D., Cohen, M. M., Jenkins, E. C., Kowal, D., Simpson, J. 1. (1989) Evaluation and development of a system for automated preparation of blood specimens for cytogenetic analysis. In "Automation of cytogenetics", Eds. C. Lundsteen and J. Piper, pp. 149-173. Springer-Verlag, Berlin kHeidelberg New York.

5. Lundsteen, C., Gerdes, T., Maahr, J., Philip, J. (1987) Clinical performance of a system for semiautomated chromosome analysis. Am J Hum Genet 41: 493-502.

6. Lundsteen, C. and Granum, E. (1976) Microphotometry of banded human chromosomes III. Scanning on photographic negatives. Clin Genet 10: 33-40.

7. Siegel, S. and Castellan, N. J. (1988) Nonparametric statistics for the behavioral sciences. McGraw-Hill Book Company, Skingapore.

8. Altman, D. G. (1991) Practical statistics for medical research. Chapman and Hall, London Tokyo Melbourne Madras.

9. Lundsteen, C., Philip, J., Granum, E. (1980) Quantitative analysis of 6985 digitized trypsin G-banded human metaphase chromosomes. Clin Genet 18: 355-370. 